\section{Nosocomial Outbreak of Middle East Respiratory Syndrome in a Large Tertiary Care Hospital - Riyadh, Saudi Arabia, 2015}

Hanan H. Balkhy, $\mathrm{MD}^{1}$; Thamer H. Alenazi, MD ${ }^{1}$; Majid M. Alshamrani, $\mathrm{MD}^{1}$; Henry Baffoe-Bonnie, $\mathrm{MD}^{1}$; Hail M. Al-Abdely, $\mathrm{MD}^{3}$; Aiman El-Saed, MD, $\mathrm{PhD}^{1}$; Hussain A. Al Arbash, MD ${ }^{4}$; Zayid K. Al Mayahi, MD ${ }^{4}$; Abdullah M. Assiri, MD5; Abdulaziz bin Saeed, MD 5

Since the first diagnosis of Middle East respiratory syndrome (MERS) caused by the MERS coronavirus (MERS-CoV) in the Kingdom of Saudi Arabia in 2012, sporadic cases and clusters have occurred throughout the country (1). During June-August, 2015, a large MERS outbreak occurred at King Abulaziz Medical City, a 1,200-bed tertiary-care hospital that includes a 150-bed emergency department that registers 250,000 visits per year.

In late June 2015, approximately 3 months after the last previously recognized MERS case in the hospital, a man aged 67 years with multiple comorbidities (diabetes, hypertension, congestive heart failure, and a history of coronary artery bypass graft surgery) and a 10-day history of fever and cough was evaluated in the emergency department (Figure). The patient had no identified exposure to camels. A nasopharyngeal swab from the patient tested positive for MERS-CoV by reverse transcription-polymerase chain reaction (RT-PCR) (2). The patient was admitted and died in the hospital after 31 days. Although this patient's hospitalization overlapped with the onset of subsequent hospital-associated MERS cases, no direct links between this first case and any of the subsequent cases were identified.

Approximately 3 weeks after the first patient's admission, a second patient, a man aged 56 years, with multiple comorbidities (diabetes with hypothyroidism, coronary artery disease, and hypertension with a history of coronary artery bypass surgery) and a history of camel exposure was evaluated in the emergency department for fever, cough, and shortness of breath. His nasopharyngeal specimen tested positive for MERS-CoV by RT-PCR. Three additional cases of MERS were epidemiologically linked to this patient's illness during his first week of hospitalization, including infections in two health care workers from the emergency department. An outbreak investigation was conducted by the hospital's infection control program to identify risk factors for infection and to develop and implement control measures. A suspected MERS case was defined as the occurrence of respiratory symptoms in a person with or without documented exposure to a patient with confirmed or probable MERS infection, but without confirmation by laboratory test results. A probable case was the occurrence of respiratory symptoms in a person with history of exposure to a patient with confirmed or probable MERS infection, but with inconclusive laboratory results (such as positive results by PCR on only one of the two genomic targets). A confirmed case was a suspected or probable case that was subsequently confirmed by a positive RT-PCR test for MERS-CoV. Contacts of persons with confirmed and probable cases were screened and persons with suspected cases were tested.

A total of 130 MERS cases were detected at King Abulaziz Medical City during late June-late August. Among these cases, 81 (62\%) were confirmed and $49(38 \%)$ were probable, including $43(33 \%)$ cases in health care workers; 20 of these 43 cases (47\%) occurred in emergency department health care workers, and $23(53 \%)$ were in health care workers from other areas of the hospital. The majority of confirmed cases were linked to the emergency department. The median age of MERS patients who were health care workers was 37 years, and $77 \%$ were female; among MERS patients who were not health care workers, the median age was 66 years, and 65\% were male. Signs and symptoms included fever and one or more respiratory symptoms, primarily cough and shortness of breath. Twenty-one (16\%) asymptomatic cases were detected during contact screening, including infection in 18 health care workers. Overall, 96 (74\%) MERS patients required hospitalization, including 63 (66\%) who required intensive care management; $34(26 \%)$ patients were isolated at home. Among all 130 cases, $51(53 \%)$ died; no deaths occurred among health care workers.

On August 2, a preexisting Infectious Disease Epidemic Plan (IDEP), established by the hospital outbreak committee and based on CDC and World Health Organization guidelines $(3,4)$, was activated (Figure). The plan included strict enforcement of infection control measures, including hand hygiene, airborne and contact isolation for confirmed and probable cases, and droplet and contact isolation for suspected cases. Measures were taken to house suspected patients and confirmed/probable patients on separate wards. Because cases continued to be identified despite the hospital's status of being in level II IDEP, on August 18, the plan was escalated to the highest level, IDEP level III, which included closure of the emergency department, postponement of elective surgical procedures, and suspension of all outpatient appointments and visits. Complete evacuation of the emergency department was achieved on August 22, and was associated with a rapid decline in the number of new cases. Onset of symptoms in the last infected patient was August 28. On September 28, the end 
FIGURE. Number of cases of Middle East Respiratory Syndrome ( $N=130)$, by week of symptom onset and health care worker (HCW) status — King Abdulaziz Medical City, Riyadh, Saudi Arabia, June-August, 2015

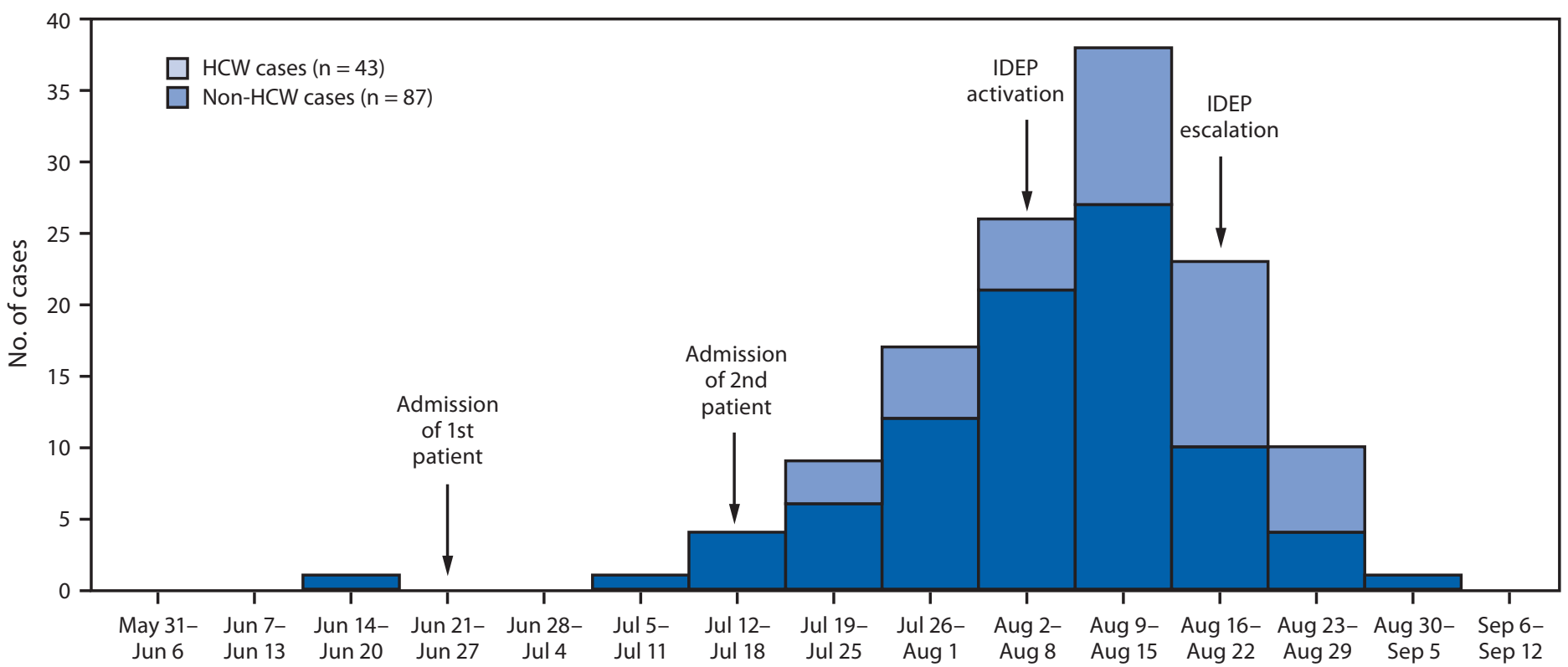

Abbreviation: IDEP = infectious disease epidemic plan.

Week of symptom onset

of outbreak was declared after the completion of two 14-day incubation periods without further identification of new cases.

This large MERS outbreak in a major tertiary-care hospital in Riyadh was thought to be related to emergency department overcrowding, uncontrolled patient movement, and high visitor traffic. The outbreak required institution of multiple measures to interrupt transmission, including almost complete shutdown of the hospital. Primary MERS cases have been linked to patients with camel exposure in previously described outbreaks (5) and exposure to camels was confirmed in three patients during the early stages of this outbreak. Escalation of the outbreak, however, was clearly linked to extended health care-related person-to-person transmission. In addition to the community transmission, four generations of hospital transmission were believed to have occurred during the outbreak. Although data are still limited, this occurrence is considered a more intense transmission than has been previously described in similar outbreaks (6). Although the outbreak was associated with considerable patient mortality, no deaths occurred among health care workers, who were younger, healthier, and had fewer comorbidities compared with patients who were not health care workers. Early recognition of cases and rapid implementation of infection control guidance is necessary to prevent health care facility-associated outbreaks of MERS-CoV.
${ }^{1}$ King Abdulaziz Medical City; Ministry of National Guard Health Affairs, Riyadh, Saudi Arabia; ${ }^{1}$ King Abdulaziz Medical City, Ministry of National Guard Health Affairs, Jeddah, Saudi Arabia; ${ }^{3}$ Ministry of Health, Riyadh, Saudi Arabia; ${ }^{4}$ Field Epidemiology Training Program, Ministry of Health, Riyadh, Saudi Arabia; ${ }^{5}$ King Fahad Medical City, Ministry of Health, Riyadh, Saudi Arabia.

Corresponding author: Hanan Balkhy, MD, 966-1-801-1111-43720, balkhyh@ngha.med.sa.

\section{References}

1. Alameer K, Abukhzam B, Khan W, El-Saed A, Balkhy H. Middle East respiratory syndrome coronavirus (MERS-Cov) screening of exposed healthcare workers in a tertiary care hospital in Saudi Arabia. Antimicrob Resist Infect Control 2015;4(Suppl 1):O57. http://dx.doi.org/10.1186/2047-2994-4-S1-O57.

2. World Health Organization. Laboratory testing for Middle East respiratory syndrome coronavirus. Interim guidance. Geneva, Switzerland: World Health Organization; 2015. http://apps.who.int/iris/bitstream/10665/176982/1/ WHO_MERS_LAB_15.1_eng.pdf?ua=1.

3. CDC. Interim infection prevention and control recommendations for hospitalized patients with Middle East respiratory syndrome coronavirus (MERS-CoV). Atlanta, GA: US Department of Health and Human Services, CDC; 2015. http://www.cdc.gov/coronavirus/mers/infectionprevention-control.html\#infection-prevention.

4. World Health Organization. Infection prevention and control of epidemicand pandemic-prone acute respiratory diseases in health care. Geneva, Switzerland: World Health Organization, 2007. http://apps.who.int/iris/ bitstream/10665/69707/1/WHO_CDS_EPR_2007.6_eng.pdf?ua=1.

5. Alraddadi BM, Watson JT, Almarashi A, et al. Risk factors for primary Middle East respiratory syndrome coronavirus illness in humans, Saudi Arabia, 2014. Emerg Infect Dis 2016;22:49-55. http://dx.doi.org/10.3201/eid2201.151340.

6. Fagbo SF, Skakni L, Chu DKW, et al. Molecular epidemiology of hospital outbreak of Middle East respiratory syndrome, Riyadh, Saudi Arabia, 2014. Emerg Infect Dis 2015;21:1981-8. http://dx.doi.org/10.3201/eid2111.150944. 